\title{
J. WARSCHAT
}

\section{Optimal control of a production-inventory system with state constraints and a quadratic cost criterion}

Revue française d'automatique, d'informatique et de recherche opérationnelle. Recherche opérationnelle, tome 19, $\mathrm{n}^{\circ} 3$ (1985), p. 275-292.

<http://www.numdam.org/item?id=RO_1985_19_3_275_0>

(C) AFCET, 1985, tous droits réservés.

L'accès aux archives de la revue « Revue française d'automatique, d'informatique et de recherche opérationnelle. Recherche opérationnelle » implique l'accord avec les conditions générales d'utilisation (http://www.numdam.org/ legal.php). Toute utilisation commerciale ou impression systématique est constitutive d'une infraction pénale. Toute copie ou impression de ce fichier doit contenir la présente mention de copyright.

\section{Numdam}

Article numérisé dans le cadre du programme

Numérisation de documents anciens mathématiques

http://www.numdam.org/ 


\title{
OPTIMAL CONTROL OF A PRODUCTION-INVENTORY SYSTEM WITH STATE CONSTRAINTS AND A QUADRATIC COST CRITERION (*)
}

\author{
by J. WARSChat $\left({ }^{1}\right)$
}

\begin{abstract}
In this paper a production-inventory system with state constraints is considered. For the unconstrained case optimal bang-bang and singular controls are derived. If the constraint is active an additional boundary-control occurs. For both cases the structure of the optimal control is obtained and it is shown that the control is discontinuous at junction points.
\end{abstract}

Keywords: Minimum principle; state constraints; production-inventory systems.

Résumé. - Dans ce travail on considère un système de stockage de la production soumis à des contraintes d'état. Dans le cas où aucune contrainte ne s'exerce, une commande optimale est dérivée, qui consiste d'une part "bangbang " et d'autre part singulier. Lorsqu'il y a une contrainte active, une commande en marge supplémentaire se met en place. Dans les deux cas, on obtient la structure d'une commande optimale et il est démontré que la commande est discontinue aux points de jonction.

Mots clés : principe de minimum; contrainte d'état; stockage.

\section{INTRODUCTION}

Since the production-inventory model of Holt et al. [7], which developed optimal production planning policies by the application of variational calculus, a number of control theoretic papers appeared (for survey see Sethi [12] and Feichtinger [3]) on this topic. Some of these models incorporate restrictions on the control as well as on the state variables, thereby the models consist of an inventory and an "inertless" production. Recently Warschat, Wunderlich [13, 14] derived time-optimal and output-maximal policies, respectively, for cascaded systems consisting of inventories and time-delayed productions in the presence of control and state constraints. In this paper we are dealing with a single production-inventory system of order two with control and state constraints and a quadratic cost criterion. Thus not only regular and bounded but also singular arcs may occur.

(*) Received March 1984.

This work was supported by the Stiftung Volkswagenwerk.

(1) Fraunhofer-Institute for Industrial Engineering (I.A.O.), Silberburgstr. 119 A, 7000 Stuttgart 1 .

R.A.I.R.O. Recherche opérationnelle/Operations Research, 0399-0559/85/03 275 18/\$3.80

(C) AFCET-Gauthier-Villars. 


\section{THE MODEL}

Consider a plant consisting of a production unit and an inventory for finished goods. The rate of change in inventories is equal to the difference between actual production rate $p(t)$ and sales rate $s(t)$ :

$$
d i(t) / d t=p(t)-s(t), \quad i(0)=i_{0} .
$$

The production plant is assumed to react only with a certain time-delay on a change of the desired production rate $p_{d}(t)$. So we get the following differential equation ( $c f$. Bradshaw and Erol [2]):

$$
d p(t) / d t=\alpha\left(p_{d}(t)-p(t)\right), \quad p(0)=p_{0},
$$

with the time delay $\alpha \in \mathbb{R}^{+}$.

Commonly $p_{d}(t)$ cannot be chosen arbitrarily but only within certain limits, say:

$$
-1 \leqq p_{d}(t) \leqq 1, \quad t \in[0, T],
$$

where $T$ denotes the planning horizon.

Moreover let us assume:

$$
p(t) \geqq 0, \quad t \in[0, T],
$$

such that backorder from the inventory is excluded.

To avoid an overtension of the system, the actual production rate is restricted:

$$
p(t) \leqq p_{\max }<1, \quad t \in[0, T] .
$$

For micro-economic problems, if the plant represents a small part of a company, especially in the case of short term planning, the sales forecasts concerning the company are broken down by the production plan where the sales rate for the plant is determined as fixed number of goods, which must be delivered at the end of the planning horizon $T$. So we have instead of a function $s(t)$ the endcondition:

$$
i(T)=i_{T}
$$

Choosing the state variables as follows:

$$
\begin{gathered}
x_{1}(t):=i(t)-i_{T}, \\
x_{2}(t):=p(t),
\end{gathered}
$$

R.A.I.R.O. Recherche opérationnelle/Operations Research 
we get the initial conditions from (1) and (2):

$$
\left.\begin{array}{c}
x_{1}(0)=i_{0}-i_{T}=:-\chi, \quad \chi \in \mathbb{R}^{+} \\
x_{2}(0)=p_{0}, \quad \text { where we assume } p_{0}=0,
\end{array}\right\}
$$

the endcondition from (6):

$$
\left.\begin{array}{c}
x_{1}(T)=0 \\
x_{2}(T): \text { free, }
\end{array}\right\}
$$

and with the desired production rate $p_{d}(t)$ as control variable $u(t)$ the system:

$$
\dot{\mathbf{x}}(t)=\mathbf{A} \mathbf{x}(t)+\mathbf{b} u(t),
$$

with the state matrix:

$$
\mathbf{A}=\left[\begin{array}{cc}
0 & 1 \\
0 & -\alpha
\end{array}\right]
$$

and the control vector:

$$
\mathbf{b}=\left[\begin{array}{l}
0 \\
\alpha
\end{array}\right] \quad \text { where }|u(t)| \leqq 1 .
$$

The objective is now to drive the system (11) from (9) to (10) with minimal production and inventory costs, both assumed to depend quadratically upon the respective state variables, which leads to the cost functional:

$$
J(u)=\frac{1}{2} \int_{0}^{T} \mathbf{x}^{T}(t) \mathbf{C x}(t) \mathrm{dt} \rightarrow \min ,
$$

where $\mathbf{C}$ denotes a $2 \times 2$ positive semidefinite weighting matrix. Additionally we assume $\mathbf{C}$ to be diagonal $\mathbf{C}:=\operatorname{diag}\left[c_{1}, c_{2}\right]$, with the constant $c_{1}$ weighting the deviation from the desired inventory $\chi$ and $c_{2}$ for the costs producing a good.

\section{OPTIMAL POLICIES FOR THE UNCONSTRAINED MODEL}

In order to get optimal control policies for (13) with the side conditions (9), (10), (11) and (12) we apply the following minimum principle (Boltjanski [1]):

For each optimal solution $\mathbf{x}(t), u(t)$ of the problem (9) to (13) there exists and adjoint vector $\lambda(t) \in \mathbb{R}^{2}$, a real number $\lambda_{0}$ and a real number $\sigma \geqq 0$ satisfying (14) to (17): 
with the Hamiltonian:

$$
H(\mathbf{x}(t), u(t), \lambda(t)):=\lambda^{T}(t) \mathbf{A} \mathbf{x}(t)+\lambda^{T}(t) \mathbf{b} u(t)+\frac{1}{2} \lambda_{0} \mathbf{x}^{T}(t) \mathbf{C} \mathbf{x}(t)
$$

we get:

$$
\begin{aligned}
\dot{\lambda}(t):=-H_{\mathbf{x}}= & -\mathbf{A}^{T} \lambda(t)-\lambda_{0} \mathbf{C x}(t), \\
\lambda(T) & =\sigma Q_{\mathbf{x}}(\mathbf{x}(T)),
\end{aligned}
$$

where $Q$ denotes a function $Q: \mathbb{R}^{2} \rightarrow \mathbb{R}$, representing the terminal manifold (10).

These relations hold for the problems fulfilling the Slater-condition (see Girsanov [5]), which is assumed in the present case. This includes the regularity of the problem such that we can set $\lambda_{0}=1$. The minimum principle takes the form:

$$
H(\mathbf{x}(t), u(t), \lambda(t))=\min _{|u| \leqq 1} H(\mathbf{x}(t), u, \lambda(t))
$$

As switching function we define:

$$
H_{u}:=\varphi(t)=\lambda^{T}(t) \mathbf{b}
$$

In the regular case (18) posses only isolated zeros and we derive a bangbang control from (17):

$$
u(t)=\left\{\begin{array}{cc}
-1 & \text { if } \quad \varphi(t)>0 \\
1 & \text { if } \quad \varphi(t)<0
\end{array}\right.
$$

Since $u(t)$ enters $H($.$) linearly, \varphi(t) \equiv 0$ may hold over a nonzero interval:

$$
I^{s}:=\left[t_{1}^{s}, t_{2}^{s}\right] \subset[0, T],
$$

which is called a singular arc.

For the following derivations it is assumed that the generalized LegendreClebsch (GLC) condition holds on an optimal singular trajectory:

$$
(-1)^{q} \frac{\partial}{\partial u}\left(\frac{d^{2 q}}{d t^{2 q}} \varphi(t)\right) \geqq 0
$$

with inequality (strengthened GLC-condition). 
To get an expression for $u(t)$ one has to evaluate the total derivatives of $\varphi(t)$ along the trajectories of (11) and set them equal to zero (Gabasov, Kirillova [4]):

$$
\frac{d^{m}}{d t^{m}} \varphi(t)=0, \quad m=1,2, \ldots
$$

The value of the index where $u$ appears explicity in (21) must be even (McDanell, Powers [11]), $m=2 q$ :

$$
\varphi^{(2 q)}(t):=a(\mathbf{x}, \lambda)+b(\mathbf{x}, \lambda) u=0,
$$

with $b(\mathbf{x}, \lambda) \neq 0$. The integer $q$ is called the order of the singular control.

Thus we derive:

$$
u(t)=-a(\mathbf{x}, \lambda) / b(\mathbf{x}, \lambda)
$$

\section{(a) Regular case}

Let us consider the regular case in phase plane (fig. 1). From (11) we get:

$$
d x_{1} / d x_{2}=-x_{2} / \alpha\left(x_{2}-u\right) .
$$

Integrating (25) with respect to (12) the following trajectories turn out:

$$
x_{1}=-\frac{1}{\alpha} x_{2}-\frac{1}{\alpha} \ln \left|x_{2} \mp 1\right|+\frac{1}{\alpha} x_{20} \pm \frac{1}{\alpha} \ln \left|x_{20} \mp 1\right|+x_{10}\left\{\begin{array}{l}
u=+1, \\
u=-1 .
\end{array}\right.
$$

From the structure of $\mathbf{A}$ follows that the set of stable points coincide with the $x_{1}$-axis. Another evident fact is that: starting within the stripe $\Sigma_{0}:=\left\{\mathbf{x}|| x_{2} \mid \leqq 1\right\}$ we can never leave $\Sigma_{0}$ with an admissible control.

Now we turn to the time dependent behaviour of (11).

Integrating the second equation one gets:

$$
x_{2}(t)=\int_{t_{0}}^{t} e^{-\alpha(t-\tau)} \alpha u(\tau) d \tau+e^{-\alpha\left(t-t_{0}\right)} x_{2}\left(t_{0}\right) .
$$

Taking (12) into account it turns out with $x_{2}\left(t_{0}\right)=0$ :

$$
x_{2}(t)= \pm\left(1-e^{\alpha\left(t_{0}-t\right)}\right) \quad \text { for } \quad u(t)=\left\{\begin{array}{l}
+1 \\
-1
\end{array}\right.
$$


Integrating $x_{2}(t)$ we have as solution for $x_{1}(t)$ :

$$
\begin{gathered}
x_{1}(t)=x_{1}\left(t_{0}\right) \pm\left(\frac{1}{\alpha} e^{\alpha\left(t_{0}-t\right)}+\left(t-t_{0}\right)-\frac{1}{\alpha}\right), \\
u(t)=\left\{\begin{array}{l}
+1, \\
-1 .
\end{array}\right.
\end{gathered}
$$

With (28) and (29) we have the candidates for the bang-bang controlled part of the optimal trajectory. In the next section the singular arc candidates are worked out.

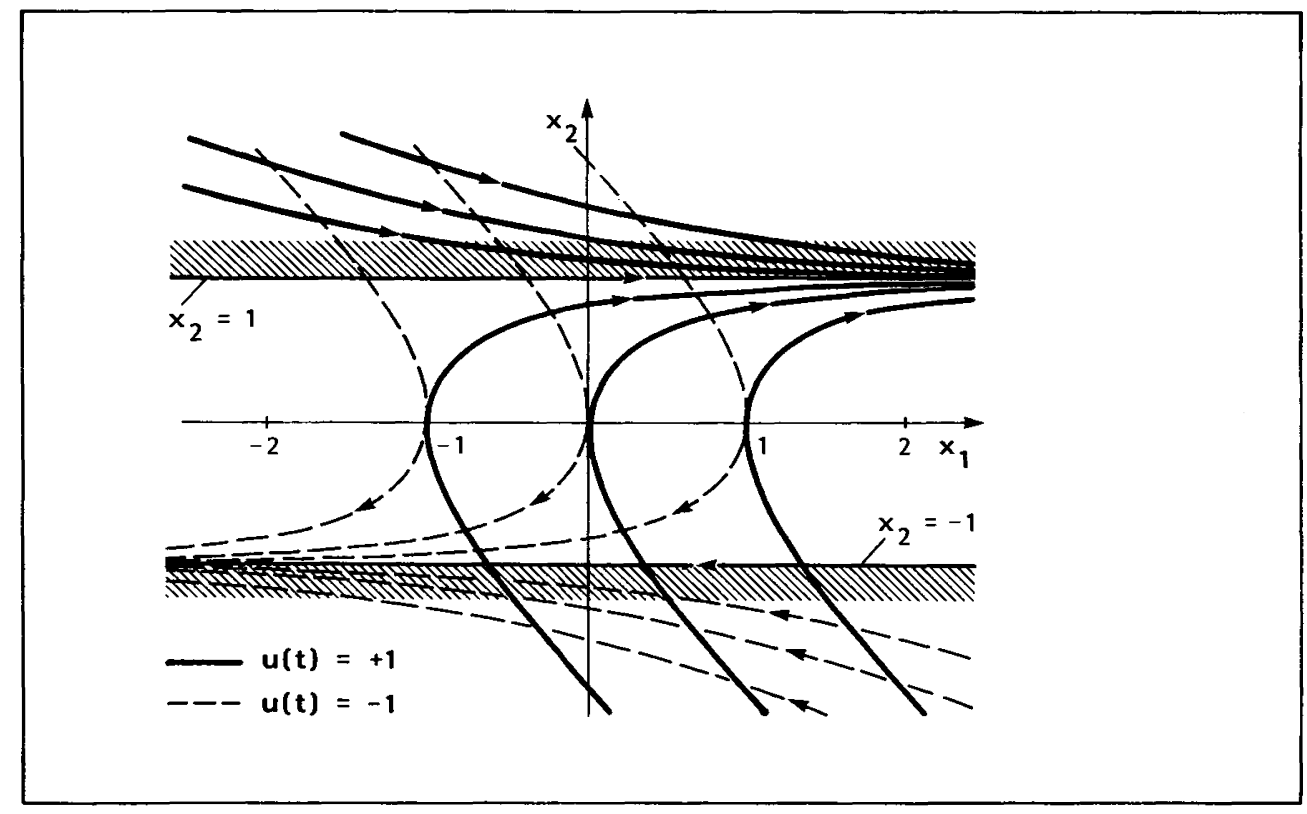

Figure 1. - Trajectories for the regular case.

(b) Singular case

The switching function follows from (18):

$$
\varphi(t)=\alpha \lambda_{2}(t) .
$$

Applying (22) we see that $q=1$ :

$$
\left.\begin{array}{l}
\varphi(t)=0, \\
\dot{\varphi}(t)=0, \\
\ddot{\varphi}(t)=0 .
\end{array}\right\}
$$

R.A.I.R.O. Recherche opérationnelle/Operations Research 
From (15), (30) and (31) we get:

$$
u^{s}(t)=\frac{c_{1}}{\alpha c_{2}} x_{1}(t)+x_{2}(t) .
$$

The substitution of (32) into (11) leads to the singular system:

$$
\dot{\mathbf{x}}^{s}(t)=\mathbf{A}^{s} \mathbf{x}^{s}(t)
$$

with:

$$
\mathbf{A}^{s}=\left[\begin{array}{ll}
0 & 1 \\
c & 0
\end{array}\right], \quad c:=c_{1} / c_{2}
$$

Remark: $\mathbf{A}^{s}$ depends no longer upon $\alpha$.

From:

$$
d x_{1} / d x_{2}=x_{2} / c x_{1},
$$

we derive:

$$
c\left(x_{1}^{2}-x_{10}^{2}\right)=x_{2}^{2}-x_{20}^{2},
$$

which yields to the phase diagram shown in figure 2 .

Obviously the singular control is not admissible in the entire phase plane because of (5). Satisfying this relation, only a stripe in the phase plane remains for admissible singular controls:

$$
\left|\frac{c}{\alpha} x_{1}+x_{2}\right| \leqq 1,
$$

which gives:

$$
\left.\begin{array}{c}
x_{2} \leqq 1-\frac{c}{\alpha} x_{1}, \\
x_{2} \geqq-1-\frac{c}{\alpha} x_{1} .
\end{array}\right\}
$$

Regarding the factor $-c / \alpha$, which is equivalent to the slope of the limiting straight lines [equalities in (37)], we can see that low production costs $c_{2} \rightarrow 0$ leads to the coincidence of the straight lines with the $x_{2}$-axis. There remains no possibility to apply a singular control. This is in accordance with the GLC-condition (21), which holds in its strengthened form only for $c_{2}>0$. If vol. $19, \mathrm{n}^{\circ} 3$, août 1985 


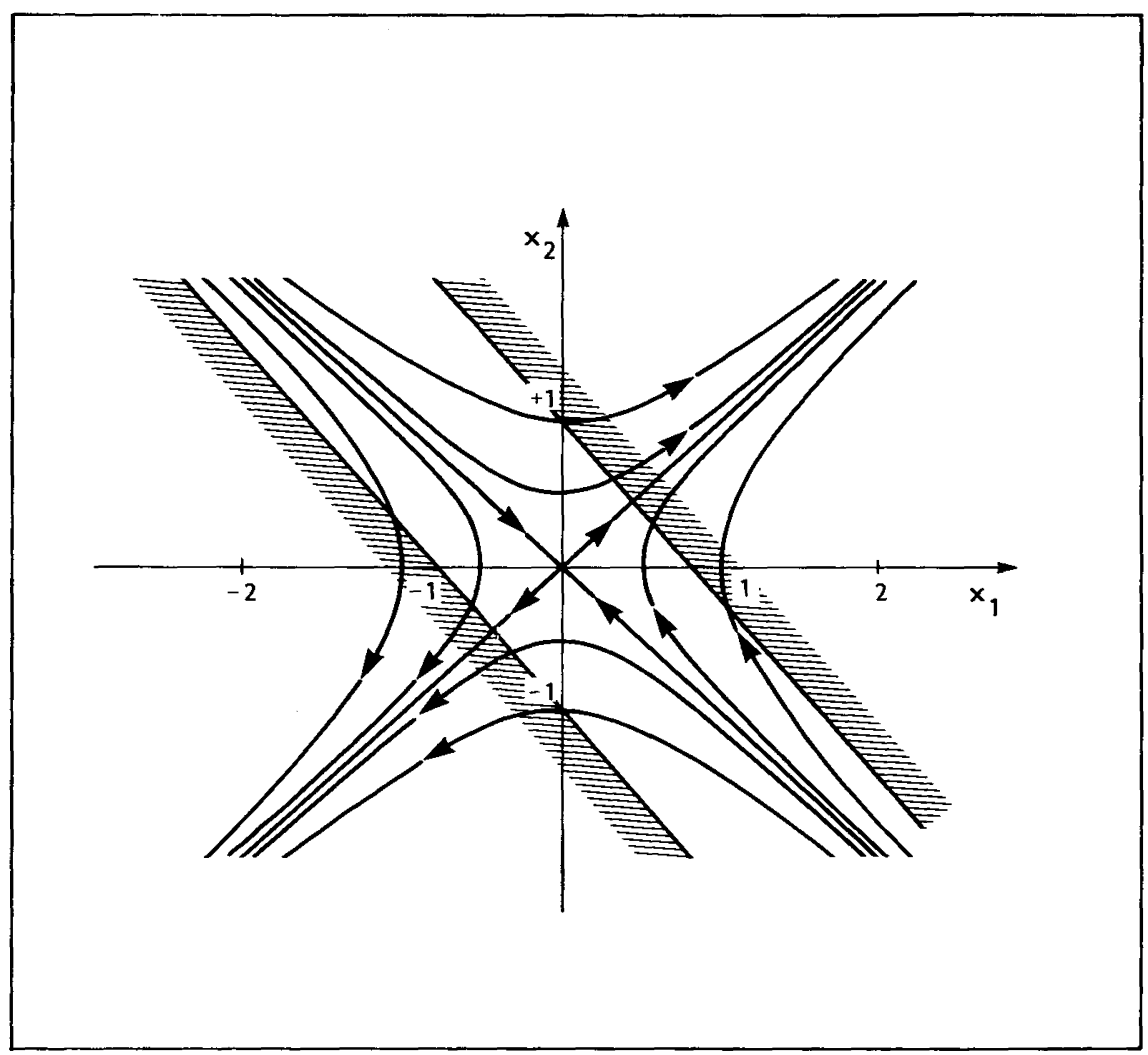

Figure 2. - Trajectories of the singular system.

we neglect on the other hand the inventory costs $c_{1}=0$, the straight lines become horizontal and it follows from (35) that the phase curves are horizontal lines too, $x_{2}=$ Const.

The solution of (33) is derived by calculating the state transition matrix (Kailath [9]):

$$
\psi(t):=\mathbf{X} e^{\Gamma t} \mathbf{X}^{-1},=
$$

where $\mathbf{X}$ denotes the modal matrix and $\boldsymbol{\Gamma}$ is the diagonal matrix of the eigenvalues $\gamma$. From the characteristical polynom the eigenvalues are calculated as:

$$
\gamma_{1,2}= \pm \sqrt{c}
$$


The modal matrix becomes:

$$
\mathbf{X}=\left[\begin{array}{cc}
1 & 1 \\
\sqrt{c} & -\sqrt{c}
\end{array}\right]
$$

As state transition matrix finally we obtain:

$$
\boldsymbol{\Psi}\left(t-t_{0}\right)=\left[\begin{array}{cc}
\cosh \left(\sqrt{c}\left(t-t_{0}\right)\right) & \frac{1}{\sqrt{c}} \sinh \left(\sqrt{c}\left(t-t_{0}\right)\right) \\
\sqrt{c} \sinh \left(\sqrt{c}\left(t-t_{0}\right)\right) & \cosh \left(\sqrt{c}\left(t-t_{0}\right)\right)
\end{array}\right] .
$$

With (41) we are able to compute the candidates for the singular arc by:

$$
\mathbf{x}(t)=\psi\left(t-t_{0}\right) \mathbf{x}\left(t_{0}\right) .
$$

Now we turn to the synthesis of the optimal trajectory.

\section{(c) Synthesis of the optimal trajectory}

The synthesis problem of optimal trajectories can be regarded from two points of view, from a local and from a global one. From local considerations we can derive the behaviour of the control function $u(t)$ at junction points of regular and singular subarcs (McDanell, Powers [11]). The global investigation yields the structure of the problem, i. e. the sequence of bang-bang and singular subarcs. In default of a general analytic solution of this problem, only numerical methods or special, problem dependent, analytical methods remain. In this chapter we shall give the structure of our problem by geometrical considerations and we shall show that the behaviour of the control $u(t)$ can be stated globally for our problem.

Let us formulate the following theorem to describe the structure of the optimal control:

Theorem 1: Let $x_{s}^{*}(T)=(1 / \alpha) e^{-T}+T-(1 / \alpha)-\chi$ denote the maximal $x_{1^{-}}$ coordinate reachable from $\mathbf{x}(0)=[-\chi, 0]^{T}$ and let $t^{s}$ denote the junction point of a regular and a singular arc of an optimal trajectory. Then:

(1) if $x_{s}^{*}(T)<0$, there exists no solution;

(2) if $x_{s}^{*}(T)=0$, the endcondition $x_{1}(T)=0$ is reached with $u(t) \equiv 1$, $t \in[0, T)$;

(3) if $x_{s}^{*}(T)>0$, the terminal manifold is reached by:

$$
u(t)=\left\{\begin{array}{cr}
1, & 0 \leqq t<t^{s}, \\
\left.u^{s}(\mathbf{x}(t))\right), & t^{s} \leqq t \leqq T .
\end{array}\right.
$$

vol. $19, \mathrm{n}^{\circ} 3$, août 1985 
Proof: The problem has a solution if $x_{1}(T)=0$ lies in the set attainable from $\mathbf{x}(0)$ by an admissible control. The boundary point of this set with respect to the $x_{1}$-coordinate is given by the maximal distance between $x_{1}(0)$ and $x_{1}(T)$. This leads to an optimization problem for the system (9) to (12) with the new optimization criterion:

$$
\widetilde{J}(u):=-x_{1}(T) \rightarrow \min .
$$

The Hamiltonian becomes:

$$
\tilde{H}:=\tilde{\lambda}^{T}(t) \mathbf{A} \mathbf{x}(t)+\tilde{\lambda}^{T} \mathbf{b} u(t)
$$

and as switching function we get:

$$
\tilde{\varphi}(t):=\alpha \tilde{\lambda}_{2}(t) .
$$

The adjoint system takes the form:

$$
\left.\begin{array}{cc}
\tilde{\lambda}_{1}(t)=0, & \tilde{\lambda}_{1}(T)=1, \\
\tilde{\lambda}_{2}(t)=\alpha \tilde{\lambda}_{2}-1, & \tilde{\lambda}_{2}(T)=0,
\end{array}\right\}
$$

which yields:

$$
\begin{gathered}
\tilde{\lambda}_{1}(t) \equiv 1, \quad t \in[0, T], \\
\tilde{\lambda}_{2}(t)=\frac{1}{\alpha}\left(e^{\alpha t}-e^{\alpha T}\right) .
\end{gathered}
$$

Since $\tilde{\lambda}_{2}(t)$ is negative over the whole interval $[0, T)$, it turns out from (19) and (45): $u(t) \equiv 1, t \in[0, T)$.

With $u(t) \equiv 1, t \in[0, T)$ we get from (29) with $t_{0}=0$ and $x_{1}\left(t_{0}\right)=-\chi$ :

$$
x_{1}^{*}=\frac{1}{\alpha} e^{-\alpha T}+T-\frac{1}{\alpha}-\chi .
$$

Thus, if $x_{s}^{*}(T)<0$ there exists no solution, which proves part (1). If $x_{s}^{*}(T)=0$, then $u(t) \equiv 1, t \in[0, T)$ holds, which proves part (2). If $x_{s}^{*}(T)>0$, a singular subarc with $|u(t)|<1$ occurs. And we know that it is optimal if it is an admissible solution, because of (21) (see Gabasov, Kirillova [4]).

At the point $[-\chi, 0]^{T}$ the gradient of the singular trajectory is $[-2 c \chi, 0]^{T}$, i. e. it is directed in an inadmissible region with respect to (4). The same fact 
holds for $u(t)=-1$. Therefore we have $u(t)=1,0 \leqq t<t^{s}$, and $u^{s}(t), t^{s} \leqq t \leqq T$, where the switching point $t^{s}$ is derived from (28), (29), (41) and (42):

$$
\begin{gathered}
x_{1}\left(t^{s}\right)=\frac{1}{\alpha} e^{-\alpha t^{s}}+t^{s}-\frac{1}{\alpha}-\chi \\
x_{2}\left(t^{s}\right)=1-e^{-\alpha t^{s}} \\
F\left(t^{s}\right):=\cosh \left(\sqrt{c}\left(T-t^{s}\right)\right) x_{1}\left(t^{s}\right)+\frac{1}{\sqrt{c}} \sinh \left(\sqrt{c}\left(T-t^{s}\right)\right) x_{2}\left(t^{s}\right)=0 .
\end{gathered}
$$

Thus we have an implicit function where we seek for a $\chi$ satisfying (3), $\alpha$ and $T$ fixed, a $t^{s} \in(0, T)$.

It can be easily seen that:

$$
\begin{aligned}
& \lim _{t^{s} \rightarrow 0} F\left(t^{s}\right)<0, \\
& \lim _{t^{s} \rightarrow T} F\left(t^{s}\right)>0 .
\end{aligned}
$$

Hence a zero exists.

In order to show the uniqueness of $t^{s}$ we use the fact that $F\left(t^{s}\right)$ is a monotonous function.

The derivative of (51) with respect to $t^{s}$ yields:

$$
F_{t^{s}}(.)=\sinh \left(\sqrt{c}\left(T-t^{s}\right)\right)\left(\frac{1}{\sqrt{c}} \frac{d x_{2}\left(t^{s}\right)}{d t^{s}}-\sqrt{c} x_{1}\left(t^{s}\right)\right) .
$$

The second term is always positive bearing in mind our assumptions. As a consequence $F_{t^{s}}()=$.0 if $\sinh \left(\sqrt{c}\left(T-t^{s}\right)\right)=0$. But this holds only for $t_{0}^{s}=T$, which contradicts (3). Therefore we have:

$$
F_{t^{s}}\left(t^{s}\right)>0 \quad \text { if } \quad t^{s} \in(0, T) .
$$

From (49), (50) and (51) we can compute the switching time $t^{s}$ and thus we are able to compute the control $u(t)$ and the state variables $x_{1}(t), x_{2}(t)$, $t \in[0, T]$.

This completes the proof.

Let us now investigate the behaviour of $u(t)$ at the junction point. From McDanell, Powers ([11], Thm. 3) we have a local condition for the continuity of $u\left(t^{s}\right)$. In our case we can derive a global condition: 
THeOREM 2: Let $l \geqq 0$ denote the lowest order derivative of $u(t)$, which is discontinuous at $t^{s}$. Then $l=0$ for all $t^{s} \in(0, T)$.

Proof: Suppose $l>0$. Then:

$$
u_{\text {regular }}\left(t^{s}\right)=u^{s}\left(t^{s}\right), \quad t^{s} \in(0, T) .
$$

This yields:

$$
1=\frac{c}{\alpha} x_{1}(t)+x_{2}(t)
$$

which is exactly the expression for the upper limiting straight line of the admissible singular region. Thus within the admissible stripe $u\left(t^{s}\right)$ is discontinuous, i. e. $l=0$.

Example 1: Figure 3 shows the history of the production rates and inventory with $\alpha=0.8, c_{1}=c_{2}=1, T=4.0, \chi=1.5$. The switching point is $t^{s}=1.7007$ and the minimal costs become $J(u)=3.5565$.

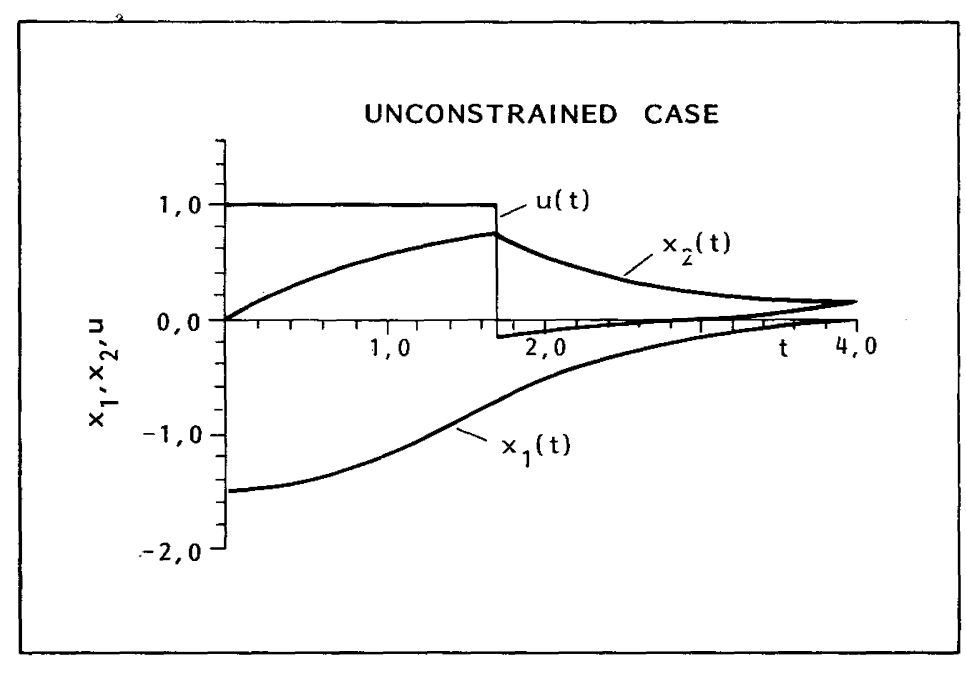

Figure 3. - Production rates and inventory for the unconstrained case.

\section{OPTIMAL POLICIES FOR THE CONSTRAINED MODEL}

Now restriction (5) comes into play:

$$
S(\mathbf{x}(t)):=x_{2}(t) \leqq p_{\max }=\beta<1, \quad t \in[0, T] .
$$

R.A.I.R.O. Recherche opérationnelle/Operations Research 
Analoguously to (20) we define an interval:

$$
I^{b}:=\left[t_{1}^{b}, t_{2}^{b}\right] \subset[0, T],
$$

called a boundary arc, where the trajectory lies on the boundary $S(\mathbf{x})$.

Because of the linearity of the Hamiltonian concerning $u(t)$ we apply Maurer's [10] version of the minimum principle, which goes back to the works of Jacobsen, Lele and Speyer [8] and of Hamilton [6]. For each optimal solution $\mathbf{x}(t), u(t)$ of the problem (9) to (13) and (57) there exists an adjoint vector $\lambda(t) \in \mathbb{R}^{2}$, a real number $\sigma \geqq 0$ and a measurable function $\eta:[0, T] \rightarrow \mathbb{R}$ with $\eta(t) S(\mathbf{x}(t)) \equiv 0, t \in[0, T]$ satisfying (59) to (62):

with the Hamiltonian:

$$
\begin{aligned}
\hat{H}(\mathbf{x}(t), u(t), \hat{\lambda}(t), \eta(t))=\hat{\lambda}^{T}(t) \mathbf{A} \mathbf{x}(t)+\hat{\lambda}^{T}(t) \mathbf{b} u(t) \\
+\frac{1}{2} \mathbf{x}^{T}(t) \mathbf{C} \mathbf{x}(t)+\eta(t) S(\mathbf{x}(t))
\end{aligned}
$$

we get:

$$
\begin{gathered}
\hat{\lambda}(t)=-\mathbf{A}^{T} \hat{\lambda}(t)-\mathbf{C} \mathbf{x}(t)-\eta(t) S_{\mathbf{x}}(\mathbf{x}(t)), \\
\hat{\lambda}(T)=\sigma Q_{\mathbf{x}}(\mathbf{x}(T)) .
\end{gathered}
$$

Setting:

$$
v(t):=\eta\left(t_{1}^{+}\right)-\eta\left(t_{1}^{-}\right),
$$

hence:

$$
\lambda\left(t_{1}^{+}\right)=\lambda\left(t_{1}^{-}\right)-v S_{\mathbf{x}}\left(\mathbf{x}\left(t_{1}\right)\right), \quad v(t) \geqq 0,
$$

where $t_{1}$ denotes the junction point between an interior arc and a boundary arc.

The conditions stated in section 3 with respect to (14) to (17) remain valid, particularly the minimum principle (17) holds also for $\hat{H}($.$) . In the following$ we omit " " if no confusion is possible. Because of the "duality" of singular and constrained problems (see McDanell, Powers [11] and Maurer [10]) we can proceed in a similar way as in section 3 .

Let $r$ denote the lowest order of the time derivative of $S(\mathbf{x}(t))$ where $u(t)$ appears explicitly, then $S(\mathbf{x}(t))$ is called of order $r$. From (57) we derive:

$$
S^{(1)}(\mathbf{x}(t))=\dot{x}_{2}(t)=-\alpha x_{2}(t)+\alpha u(t)=0, \quad t \in I^{b} .
$$


Thus:

$$
u(t)=x_{2}(t)=\beta, \quad t \in I^{b} .
$$

Remark: In order to ensure the validity of $(60)$ to $(62)$ one has to avoid that $|u(t)|=1$ for $t \in I^{b}$. But due to (57) $|u(t)|<1, t \in I^{b}$ holds.

Because $S(\mathbf{x}(t))$ is of order 1 it follows (Maurer [10], Thm. 5.6) for a junction point between a regular arc and a boundary arc: $v=0$ at $t_{1}^{b}$ and additionally the optimal trajectory contains at least one boundary arc, if the constraint is active, i. e. if the unconstrained trajectory violates (57).

Now let us proceed to the investigation of the structure concerning the constrained problem.

THEOREM 3: Let the constraint (57) be active and let $t^{b}$ and $t^{s}$ denote the junction points between an interior regular arc and $a$ boundary arc and $a$ boundary arc and an interior singular arc, respectively. Moreover let:

$$
x_{1}^{* *}(T)=v \ln (1-\beta)-v-\frac{1}{\alpha}+\beta T-\chi
$$

with:

$$
v:=\frac{1}{\alpha}(\beta-1),
$$

be the maximal $x_{1}$-coordinate reachable from $[-\chi, 0]^{T}$. Then:

(1) if $x_{1}^{* *}(T)<0$, there exists no solution;

(2) if $x_{1}^{* *}(T)=0$, the endcondition $x_{1}(T)=0$ is reached with:

$$
u(t)=\left\{\begin{array}{cc}
1, & 0 \leqq t<t^{b}, \\
\beta, & t^{b} \leqq t \leqq T
\end{array}\right.
$$

(3) if $x_{1}^{* *}(T)>0$, the terminal manifold is reached by:

$$
u(t)=\left\{\begin{array}{cc}
1, & 0 \leqq t<t^{b}, \\
\beta, & t^{b} \leqq t<t^{s} \\
u^{s}(t), & t^{s} \leqq t \leqq T .
\end{array}\right.
$$

Proof: By the same considerations as in the proof of theorem 1 we yield as optimal control for the point $[-\chi, 0]^{T}, u(0)=1$. This holds until the trajectory reaches the boundary at:

$$
t^{b}=-\frac{1}{\alpha} \ln (1-\beta) \text {. }
$$

R.A.I.R.O. Recherche opérationnelle/Operations Research 
Since the unconstrained optimal trajectory (48) violates (57) for $t \in\left[t^{b}, T\right]$, the optimal trajectory remains on $S(x(t))$. From (28), (29) and (64) one easily derives:

$$
x_{1}^{* *}(T)=v \ln (1-\beta)-v-\frac{1}{\alpha}+\beta T-\chi
$$

This proofs part (1) and (2).

In the case (3) we get from (28), (29), (41), (42) and (64):

$$
\begin{aligned}
F^{b}\left(t^{s}\right):=\cosh \left(\sqrt{c}\left(T-t^{s}\right)\right)\left(\beta t^{s}+v \ln (1-\beta)-\right. & \left.v-\frac{1}{\alpha}-\chi\right) \\
& +\sinh \left(\sqrt{c}\left(T-t^{s}\right)\right) \beta=0 .
\end{aligned}
$$

One gets:

$$
\lim _{t^{s} \rightarrow T} F^{b}\left(t^{s}\right)>0
$$

Moreover it turns out:

$$
\lim _{t^{s} \rightarrow t^{b}} F^{b}\left(t^{s}\right)<0,
$$

since for $t^{s}=t^{b}$ we have exactly the case where no constraint is active. Obviously the new $t^{s}=t^{b}$ is smaller than the $t_{0}^{s}$ of the unconstrained problem. As it was shown in theorem $1, F\left(t^{s}\right)$ depends monotonically upon $t^{s}$. Therefore $F\left(t_{1}\right)<F\left(t_{2}\right)$ if $t_{1}<t_{2}$. But $F\left(t_{0}^{s}\right)=0$ and for that reason $F^{b}\left(t^{b}\right)=F\left(t^{b}\right)<0$. Thus a zero $t_{0}^{s}$ of $F^{b}\left(t^{s}\right)$ exists. The uniqueness turns out, analogously to theorem 1 , since one can show that $F_{t^{b}}^{b}\left(t^{s}\right)>0, t^{s} \in\left(t^{b}, T\right)$. Finally we get from (67) the time $t^{s}$, which enables us together with (65), the equations (28), (29), (41) and relation (57) to compute the time history of the control and state variables. This completes the proof.

Analoguously to theorem 2 we obtain for the junction points $t^{b}$ and $t^{s}$ :

THEOREM 4: Let $t^{b}$ and $t^{s}$ be defined as in theorem 3. Let $l \geqq 0$ denote the lowest order derivative of $u(t)$ which is discontinuous at $t^{b}$ and $t^{s}$, respectively. Then $l=0$ at $t^{b}, t^{s} \in(0, T), t^{s}>t^{b}$.

Proof: Suppose $l>0$.

(a) Junction between a regular interior arc and a boundary arc. If $l>0$ :

$$
u_{\text {regular }}\left(t^{b}\right)=u^{b}\left(t^{b}\right)
$$

vol. $19, \mathrm{n}^{\circ} 3$, août 1985 
Thus: $1=\beta$, which contradicts (57).

Hence $l=0$ for all $t^{b} \in(0, T)$.

(b) Junction between a boundary arc and an interior singular arc. If $l>0$ :

$$
u^{b}\left(t^{s}\right)=u^{s}\left(t^{s}\right)
$$

Thus:

$$
\beta=\frac{c_{1}}{\alpha c_{2}} x_{1}\left(t^{s}\right)+x_{2}\left(t^{s}\right)
$$

This holds only if $x_{1}\left(t^{s}\right)=0$. But that is exactly the case where we have no singular arc, hence $l=0$, for all $t^{s} \in\left(t^{b}, T\right)$, which completes the proof.

Example 2: With the same values as in example 1 and an additional constraint $\beta=0.5$ one gets $t^{b}=0.8664, t^{s}=2.4738$ and $J(u)=3.6989$. The history of $x_{1}(t), x_{2}(t)$ and $u(t)$ is shown in figure 4 .

Remark: In the constrained case as in the unconstrained case as well the necessary conditions for optimality are also sufficient (see Girsanov [5], Thm. 16,2).

\section{INTERPRETATION OF THE RESULTS}

The production-inventory model (1), (2) is an extension of the models described by Sethi [12]. In the papers reviewed there the production rate is taken as the control variable so that production can be altered immediately. The model of Bradshaw and Erol [2] distinguishes between a desired production rate and an actual production rate. The interaction between both works like a regulator. The actual production rate follows with a certain time delay the desired rate. Such "inert" dynamic behaviour can be found for example in the field of chemical processes or in production processes with learning curves.

As a consequence of the model formulation, especially by the definition of $x_{1}(t)$ in (7), which says that we consider the deviation of the actual inventory from the inventory requested at the end of the planning horizon, and the quadratic cost criterion (13) which weights a great difference much more than a small one, we start with the maximal desired production rate (fig. 3 ).

Approaching the desired inventory level a strategy which avoids high production costs is optimal, so we produce by a singular policy. The negative desired production rate means that backlogging should be admissible, though the actual production rate remains positive. If desired backlogging should be 


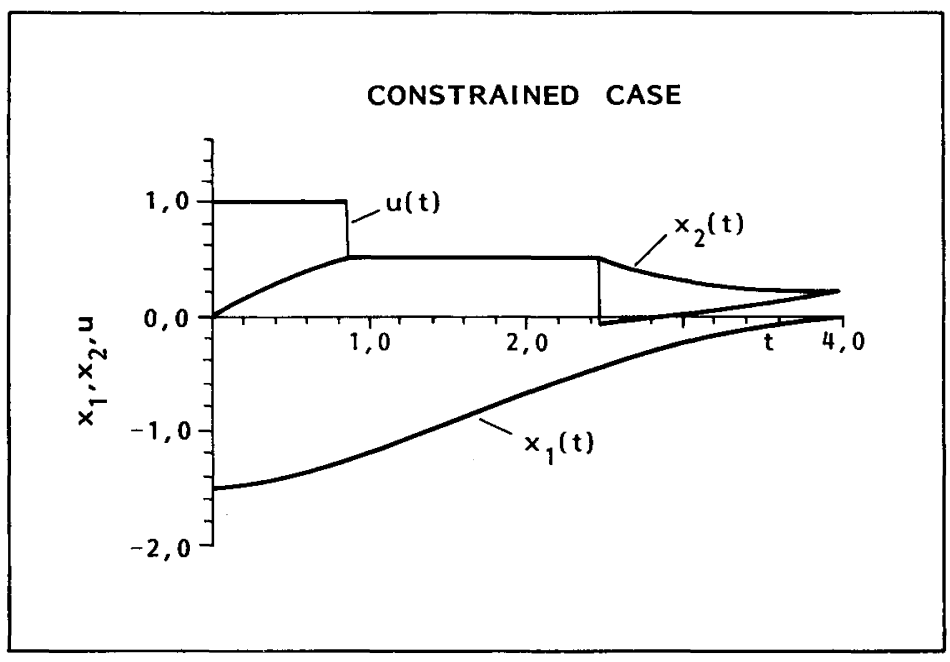

Figure 4. - Time history of the production rates and the inventory for the constrained case.

avoided the desired production rate can be restricted to $0 \leqq p_{d}(t) \leqq 1, t \in[0, T]$. Then all results are still valid, but in some cases an additional regular arc $(u(t)=0)$ at the end of the horizon is necessary to reach the target $x_{1}(T)=0$. No further problems arise if we let the actual production rate at the end time $T$ free. If we wish a fixed production rate at the end of the planning horizon only the formulation (3) guarantees the controlability of the endpoint. Without desired backlogging a production rate $x_{2}(T)=0$ would not be realizable because the origin do not belong to the interior of the admissible set of $u(t)$.

\section{CONCLUSION}

It was shown that for a state constrained production inventory system governed by a state equation of order two and subjected to a quadratic cost criterion the structure of the solution i.e, the sequence of interior regular, boundary and singular arcs can be determined uniquely. Moreover one can derive easily the optimal control for constrained as well as for unconstrained problems. More efforts should be made to investigate the behaviour of cascades consisting of a number of basic systems described in this paper.

Here the structural problem becomes more serious since the support of the phase plane technique is no longer at our disposal. 


\section{REFERENCES}

1. W. G. Boltuanski, Mathematische Methoden der optimalen Steuerung, Carl Hanser Verlag, München, 1972.

2. A. Bradshaw and Y. Erol, Control Policies for Production-Inventory Systems with Bounded Input, Int. J. Systems Sc., Vol. 11, 1980, pp. 947-959.

3. G. FeICHTINGER, Anwendungen des Maximumprinzips im Operations Research, OR Spektrum, Vol. 4, 1982, Teil 1 : No. 3, pp. 171-190, Teil 2 : No. 4, pp. 195-212.

4. R. Gabasov and F. M. Kirillova, High Order Necessary Conditions for Optimality, S.I.A.M. J. Control, Vol. 10, 1972, pp. 127-168.

5. I. V. Girsanov, Lectures on Mathematical Theory of Extremum Problems, Springer-Verlag, Berlin, Heidelberg, New York, 1972.

6. W. E. Hamilton, On Nonexistence of Boundary Arcs in Control Problems with Bounded State Variables, I.E.E.E. Trans. Autom. Control, Vol. 17, 1972.

7. C. Holt, F. Modigliani, J. Muth and H. Simon, Planning Production Inventories and Work Force, Prentice-Hall, Englewood Cliffs, 1969.

8. D. H. Jacobson, M. M. Lele and J. L. Speyer, New Necessary Conditions of Optimality for Control Problems with State-Variable Inequality Constraints, J. Math. Anal. Appl., Vol. 35, 1971, pp. 255-283.

9. Th. Kailath, Linear Systems, Prentice-Hall, Englewood Cliffs, 1980.

10. H. Maurer, On Optimal Control Problems with Bounded State Variables and Control Appearing Linearly, S.I.A.M. J. Control, Vol. 15, 1977, pp. 345-362.

11. J. P. MCDANell and W. F. Powers, Necessary Conditions for Joining Optimal Singular and Nonsingular Subarcs, S.I.A.M. J. Control, Vol. 9, 1971, pp. 161-173.

12. S. P. SETHI, A Survey of Management Science Applications of the Deterministic Maximum Principle, T.I.M.S. Studies in the Management Sciences, Vol. 9, 1978, pp. 33-67, North-Holland, Amsterdam, New York.

13. J. Warschat and H.-J. Wunderlich, Time-Optimal Control Policies for Cascaded Production-Inventory Systems with Control and State Constraints, Int. J. Systems Sc., Vol. 15, 1984, pp. 513-524.

14. J. Warschat and H.-J. Wunderlich, Output Maximization for Cascaded Production-Inventory Systems with Control and State Constraints, J.O.T.A., Submitted. 